\title{
Extending the Linearity Range of Eddy-Current Displacement Sensor With Magnetoplated Wire
}

\author{
Tsutomu Mizuno $^{1}$, Shigemi Enoki ${ }^{2}$, Toshiaki Hayashi $^{1}$, Takashi Asahina ${ }^{1}$, and Hiroki Shinagawa ${ }^{2}$ \\ ${ }^{1}$ Faculty of Engineering, Shinshu University, Nagano 380-8553, Japan \\ ${ }^{2}$ Shinkawa Sensor Technology Inc., Higashi-Hiroshima 739-0153, Japan
}

\begin{abstract}
The ratio of the linearity range $L_{\mathrm{o}}$ to the coil diameter $D$ of the ordinary eddy-current (EC) displacement sensor, $L_{\mathrm{o}} / D$, is approximately 0.48. In conventional technology, the coil diameter needs to be increased to extend the measuring range of the EC displacement sensor. In this paper, we propose the use of magnetoplated wire (MPW) for the coil winding to improve the $L_{\mathrm{o}} / D$ ratio. An MPW is a copper wire whose circumference is plated with a magnetic thin film. We discuss the physical phenomena and impedance characteristics of the MPW coil and compare them with those of a coil that uses a conventional copper wire $(C O W)$. The $L_{\mathrm{o}} / D$ values of an EC sensor with an MPW coil and with a COW coil are 1.0 and 0.48 , respectively. These results demonstrate the ability of the MPW coil to double $L_{\mathrm{o}} / D$ value over that of the COW coil.
\end{abstract}

Index Terms-Copper wire, displacement sensor, eddy current, impedance, linearity, magnetoplated wire, output voltage, quality factor, sensitivity.

\section{INTRODUCTION}

$\mathbf{T}$ THERE has been a requirement to make a coil system available with a higher $L_{\mathrm{o}} / D$ ratio ( $L_{\mathrm{o}}$ is the linearity range, $D$ is the coil diameter) to extend the measuring range of eddy-current (EC) displacement sensors. Whereas the $L_{\mathrm{o}} / D$ of the conventional copper wire (COW) coil is approximately 0.48 , we propose an MPW coil that uses magnetoplated wire (MPW) to improve the $L_{\mathrm{o}} / D$ ratio [1]. An MPW is a conductor wire whose circumference is plated with a magnetic thin film.

We examine the physical phenomena and impedance characteristics of MPW and COW coils. In addition, we demonstrate that the use of an MPW coil improves the detection sensitivity and linear measuring range because the $L_{\mathrm{o}} / D$ ratio is improved. The following points concerning EC displacement sensors with the MPW are discussed.

1) The physical phenomena of the MPW coil.

2) The improvement in detection sensitivity.

3) The extension of measuring range.

\section{Structure And Displacement Sensing PrinciPle OF EC DisPlaCEMENT SENSOR}

Fig. 1 shows the system configuration and detection principle of an EC displacement sensor. This system is composed of a measuring object, a coil, a capacitor $C_{\mathrm{p}}$ for parallel resonance, and a coupling capacitor $C_{\mathrm{s}}$. An oscillator provides a driving voltage $V$ to the coil with driving frequency $f$ tuned to the parallel resonance frequency.

The measuring object of the EC displacement sensor is usually made of a magnetic substance. Chromium molybdenum steel was used as the measuring object in this study. The magnetic flux $\Phi_{\mathrm{c}}$ is generated by the driving current $I_{\mathrm{c}}$ of the coil,

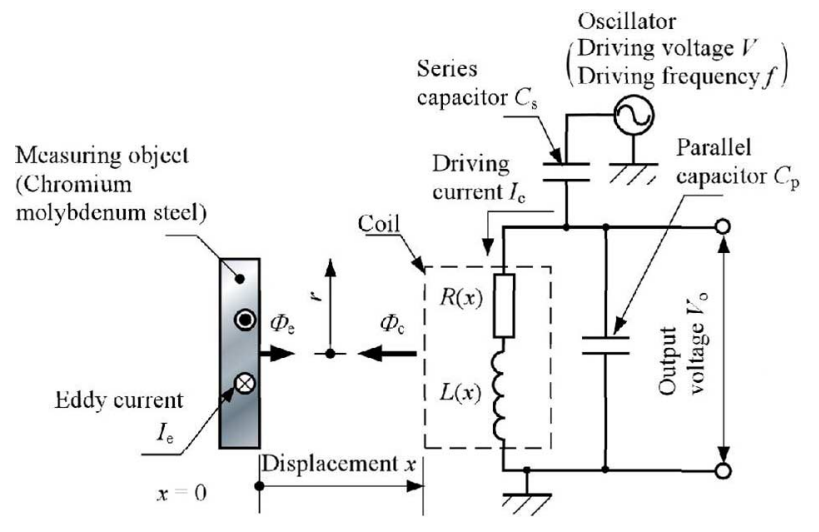

Fig. 1. System configuration and detection principle of EC displacement sensor.

and the magnetic flux $\Phi_{\mathrm{e}}$ is generated by the eddy current $I_{\mathrm{e}}$ in the measuring object. $I_{\mathrm{e}}$ changes in accordance with the displacement of the measuring object and the impedance of the coil changes as a result. The impedance of the coil converts the flux change in the coil into an output voltage $V_{\mathrm{o}}$ through the parallel resonance circuit. The construction plan in this study uses the cylindrical coordinates $(x, r)$.

$V_{\mathrm{o}}$ is expressed using the quality factor $Q(x)$ by [2]

$$
\begin{aligned}
V_{\mathrm{o}} & =\sqrt{\frac{1+Q^{2}(x)}{k^{2}+Q^{2}(x)}} \cdot V \\
Q(x) & =\omega \frac{L(x)}{R(x)} \\
k & =1+\frac{C_{\mathrm{p}}}{C_{\mathrm{s}}}
\end{aligned}
$$

where $x$ is the displacement $(\mathrm{m}), V$ is the driving voltage $(\mathrm{V})$, $\omega$ is the angular frequency $(\mathrm{rad} / \mathrm{s}), L(x)$ is the equivalent series inductance $(\mathrm{H}), R(x)$ is the equivalent series resistance $(\Omega), C_{\mathrm{p}}$ 


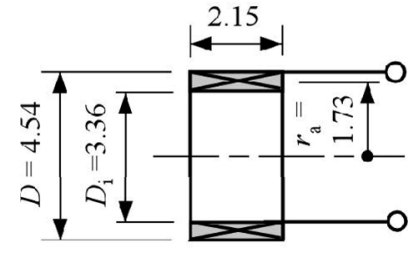

(a)

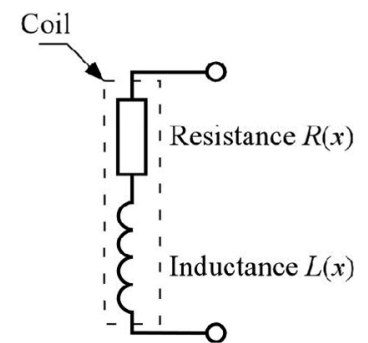

(b)
Fig. 2. Coil used for EC displacement sensor. (a) Structure (unit: mm). (b) Equivalent circuit model.

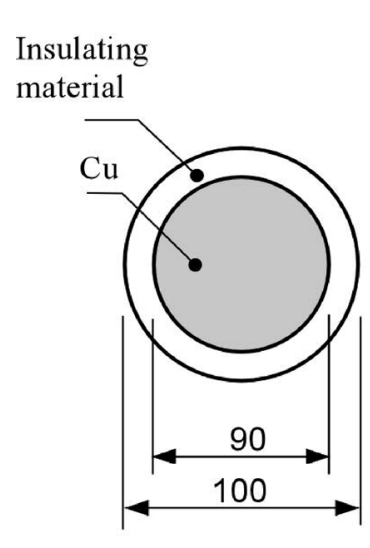

(a)

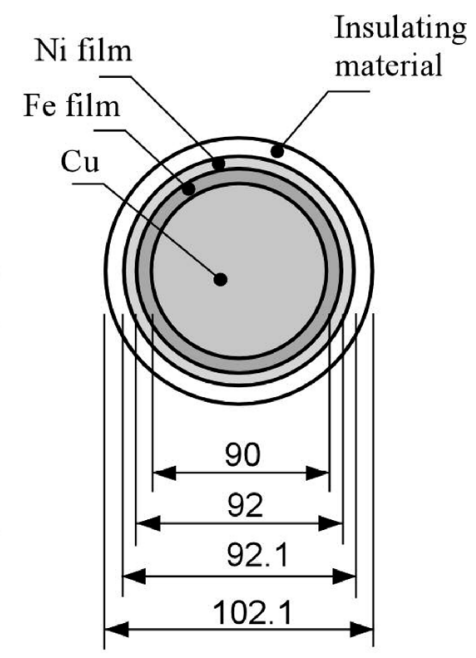

(b)
Fig. 3. Structure of conducting wire (unit: $\mu \mathrm{m}$ ). (a) COW. (b) MPW.

is the parallel capacitance $(\mathrm{F})$, and $C_{\mathrm{s}}$ is the series capacitance (F).

The output voltage $V_{\mathrm{o}}$ is expressed in terms of only $Q(x), C_{\mathrm{p}}$, and $C_{\mathrm{s}}$. The capacitor $C_{\mathrm{p}}$ determines the driving (resonant) frequency, and $C_{\mathrm{s}}$ determines the maximum value of the driving current $I_{\mathrm{c}}$ to prevent an exothermic reaction. $C_{\mathrm{p}} / C_{\mathrm{s}}$ in (3) is uniquely determined by the driving frequency and the maximum value of the driving current. Therefore, it is essential to examine $Q(x)$ against the displacement characteristics of the coil to extend the measuring range and detection sensitivity of the EC displacement sensor in a parallel resonance system [2].

\section{Physical Phenomena of MPW CoIL}

\section{A. Structure of Conductor and Coil}

Fig. 2 shows the coil used for the EC displacement sensor. The EC displacement sensor has an air core coil with coil diameter $D=4.54 \mathrm{~mm}$, average radius $r_{\mathrm{a}}=1.73 \mathrm{~mm}$, wire diameter $d=0.09 \mathrm{~mm}$, and number of turns $N=102$. The resonant frequencies of both the MPW and COW coils were measured as $10.5 \mathrm{MHz}$. The driving frequency $f=1.4 \mathrm{MHz}$, because it is sufficiently different from the resonant frequency, and the distributed capacitance of the coil, because it is sufficiently small, do not significantly influence the system characteristics. On the basis of the above conditions, the equivalent circuit shown in Fig. 2(b) was formulated for further discussion in this study.

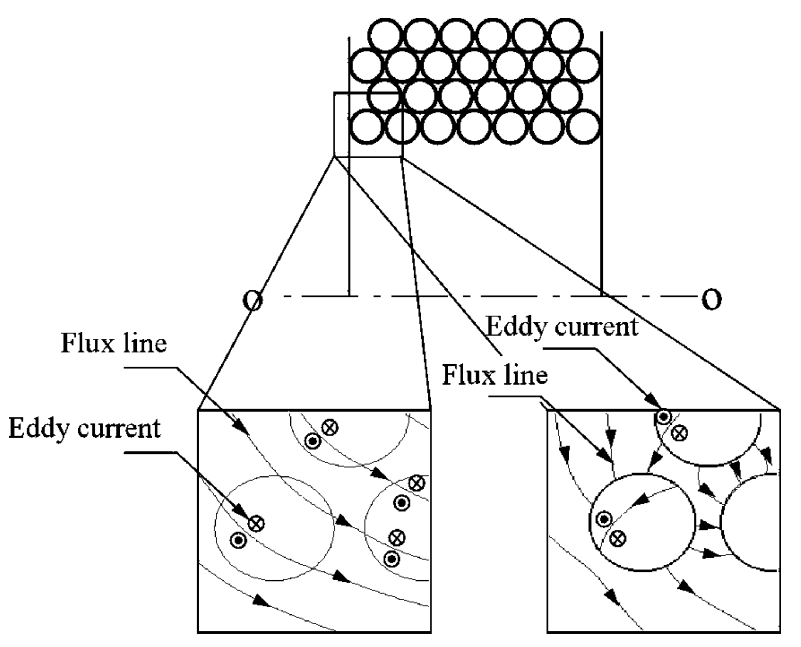

(a)

(b)

Fig. 4. Flux lines around coil analyzed by FEM. (a) COW coil. (b) MPW coil $\left(x \rightarrow \infty, f=1.4 \mathrm{MHz}, I_{\mathrm{c}}=1 \mathrm{~mA}, \mathrm{Cu}: \mu_{\mathrm{r}}=0.999991, \sigma=5.8 \times\right.$ $\left.10^{7} \mathrm{~S} / \mathrm{m}, \mathrm{Fe}: \mu_{\mathrm{r}}=200, \sigma=1.1 \times 10^{7} \mathrm{~S} / \mathrm{m}\right)$.

Fig. 3 shows the structure of the conductor wire. The MPW has a copper core plated with magnetic thin films ( $\mathrm{Fe}$ and $\mathrm{Ni}$ ) [3], [4]. The thicknesses of the Fe and Ni thin films are 1 and $0.05 \mu \mathrm{m}$, respectively, as shown in Fig. 3(b). The Ni film is plated for ease of soldering.

\section{B. Features of MPW Coil}

For this analysis, Maxwell [5], the software developed for the finite-element method (FEM), was used to clarify the physical phenomena of the MPW. The eddy current in the coil was analyzed using cylindrical coordinates with parameters $N=$ 26 turns, $D_{\mathrm{i}}=1.6 \mathrm{~mm}, D=2.24 \mathrm{~mm}$, axial length $l=$ $0.63 \mathrm{~mm}, f=1.4 \mathrm{MHz}$, and $I_{\mathrm{c}}=1 \mathrm{~mA}$. Because the thickness of the Ni thin film was $1 / 20$ that of the Fe thin film, its thickness was disregarded in this analysis. The permeability and conductivity of $\mathrm{Cu}$ were $\mu_{\mathrm{r}}=0.999991$ and $\sigma=5.8 \times 10^{7} \mathrm{~S} / \mathrm{m}$, respectively, and those of the Fe thin film were $\mu_{\mathrm{r}}=200$ and $\sigma=1.1 \times 10^{7} \mathrm{~S} / \mathrm{m}$, respectively. It was impossible to analyze the coil with 102 turns because the 2-GB memory capacity of a personal computer is insufficient for the FEM. Therefore, we analyzed the coil with 26 turns. Although the number of turns of the coil was different from those shown in Fig. 2, the effects of these differences on the physical phenomena were considered to be negligible.

Fig. 4 shows the behavior of the magnetic flux around the coil conductor, as analyzed by FEM. The proximity effect increases the resistance of the adjacent coil due to the eddy current when the flux of the adjacent coil interlinks with the coil wire [6]-[8]. Less eddy current is generated within the MPW coil owing to the shield effect of the magnetic film around the conductor wire. Therefore, the eddy-current loss within the coil decreases when the MPW coil is used.

Fig. 5 shows the current density distribution in the COW and MPW coils under FEM analysis. Because the COW coil induces more flux through the coil conductor, as shown in Fig. 4(a), more eddy current is generated in the coil. Therefore, the current density in the coil is biased more than that in the MPW coil. The resistance in the COW coil becomes larger than that in the MPW 


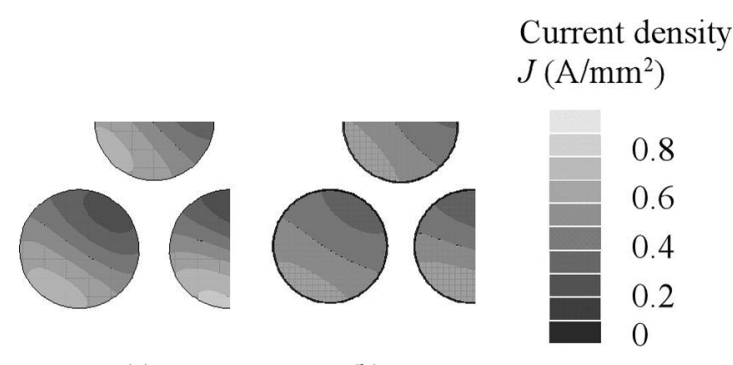

(a)

(b)

Fig. 5. Current density distribution in coil under FEM analysis $(x \rightarrow \infty, f=$ $1.4 \mathrm{MHz}, I_{\mathrm{c}}=1 \mathrm{~mA}, \mathrm{Cu}: \mu_{\mathrm{r}}=0.999991, \sigma=5.8 \times 10^{7} \mathrm{~S} / \mathrm{m}, \mathrm{Fe}: \mu_{\mathrm{r}}=200$, $\sigma=1.1 \times 10^{7} \mathrm{~S} / \mathrm{m}$ ). (a) COW coil. (b) MPW coil.

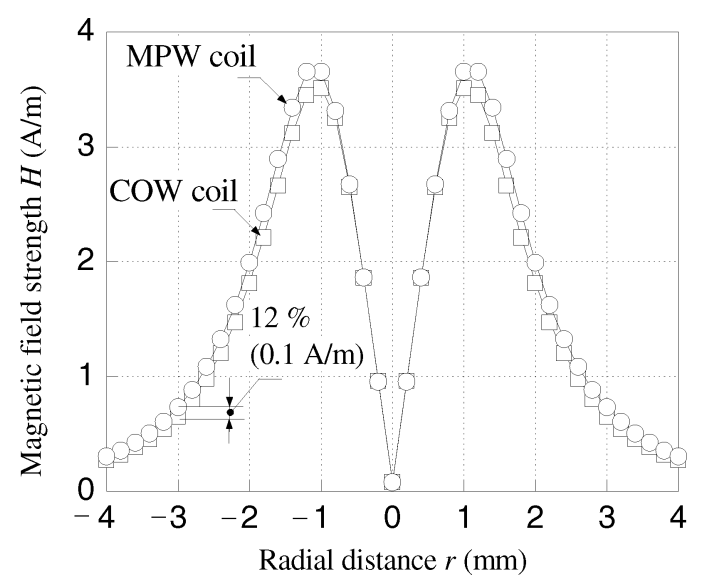

Fig. 6. Magnetic flux density on surface of measuring object obtained under FEM. ( $x=0.5 \mathrm{~mm}, f=1.4 \mathrm{MHz}, I_{\mathrm{c}}=1 \mathrm{~mA}$, measuring object: $\mu_{\mathrm{r}}=60$, $\sigma=7 \times 10^{6} \mathrm{~S} / \mathrm{m}$, diameter $12 \mathrm{~mm}$, thickness $0.5 \mathrm{~mm}, \mathrm{Cu}: \mu_{\mathrm{r}}=0.999991$, $\left.\sigma=5.8 \times 10^{7} \mathrm{~S} / \mathrm{m}, \mathrm{Fe}: \mu_{\mathrm{r}}=200, \sigma=1.1 \times 10^{7} \mathrm{~S} / \mathrm{m}\right)$.

coil due to the proximity effect caused by the heavier bias of the current density created inside the coil.

Fig. 6 shows the magnetic flux density on the surface of the measuring object under FEM. The field strength $H$ of the measuring object surface was calculated when the displacement $x$ was $0.5 \mathrm{~mm}$. The field strength of the MPW coil was larger than that of the COW coil. Therefore, it is understood that the magnetic flux affected to the measuring object more when the MPW coil was used.

\section{Frequency Characteristics of Coil}

Fig. 7 shows the impedance characteristics against frequency of the COW and MPW coils. The calculated value of $R_{\text {se }}$ due to the skin effect of the COW is shown in Fig. 7(a). The resistance due to the skin effect is calculated using [8], [9]

$$
\begin{aligned}
R_{\mathrm{se}} & =R_{\mathrm{dc}} \frac{\gamma}{2} \cdot \frac{\text { ber } \gamma \mathrm{bei}^{\prime} \gamma-\text { bei } \gamma \mathrm{ber}^{\prime} \gamma}{\mathrm{ber}^{\prime 2} \gamma+\mathrm{bei}^{\prime 2} \gamma} \\
R_{\mathrm{dc}} & =\frac{4 l}{\pi \sigma d^{2}} \quad(\Omega) \\
l & =\pi N\left(r_{\mathrm{i}}+r_{\mathrm{e}}\right) \\
\gamma & =\frac{d}{\sqrt{2} \delta} \\
\delta & =\frac{1}{\sqrt{\pi f \mu_{\mathrm{r}} \mu_{0} \sigma}}
\end{aligned}
$$

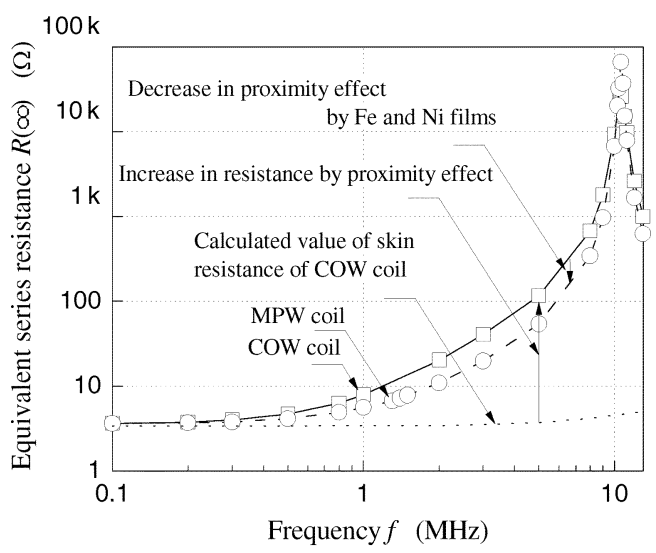

(a)

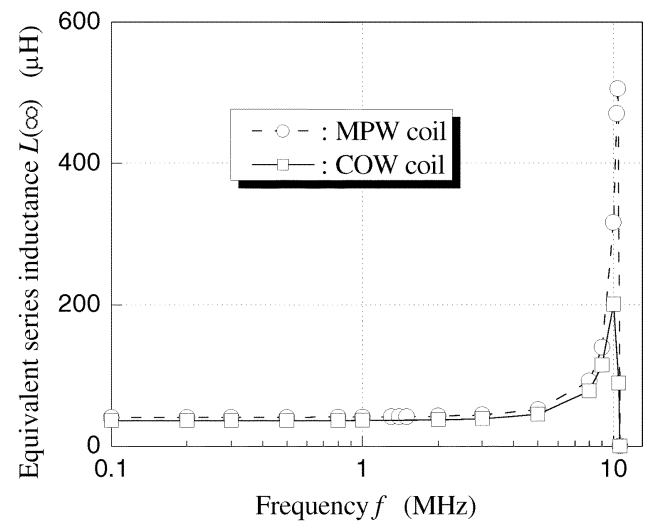

(b)

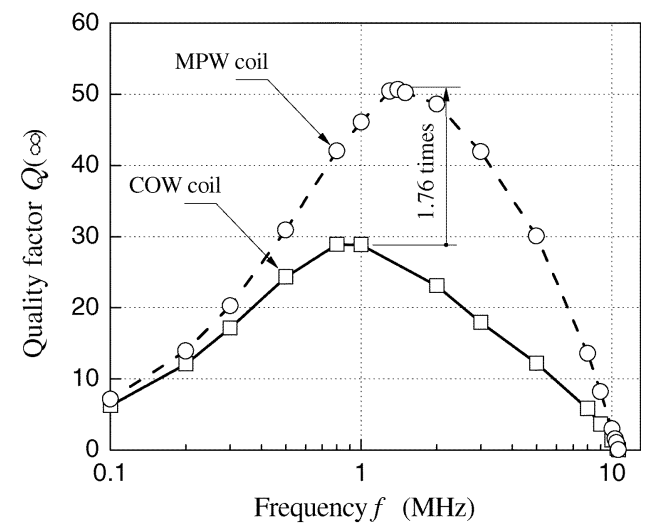

(c)

Fig. 7. Comparison of impedance characteristics versus frequency between COW and MPW coils. (a) Resistance $R(\infty)$. (b) Inductance $L(\infty)$. (c) Quality factor $Q(\infty)$.

where $R_{\mathrm{dc}}$ is the dc resistance $(\Omega)$ of the coil, ber is the zeroorder real-number Kelvin function, bei is the zero-order complex-number Kelvin function [10], $l$ is the conductor length (m), $\sigma$ is the copper conductivity $(\mathrm{S} / \mathrm{m}), d$ is the conductor wire diameter (m), $N$ is the number of turns of the winding, $r_{\mathrm{i}}$ is the inner radius of the coil $(\mathrm{m}), r_{\mathrm{e}}$ is the external form radius of the coil $(\mathrm{m}), \delta$ is the skin depth $(\mathrm{m}), f$ is the driving frequency $(\mathrm{Hz})$, $\mu_{\mathrm{r}}$ is the copper relative permeability, and $\mu_{0}$ is the vacuum permeability $\left(4 \pi \times 10^{-7}\right)(\mathrm{H} / \mathrm{m})$.

The measured value of $R(\infty)$ at $x \rightarrow \infty$ is larger than the calculated $R_{\text {se }}$, as shown in Fig. 7(a). Therefore, the cause of the increase in $R(\infty)$ is understood to be not the skin effect, but the proximity effect. $R(\infty)$ of the MPW coil was smaller than 


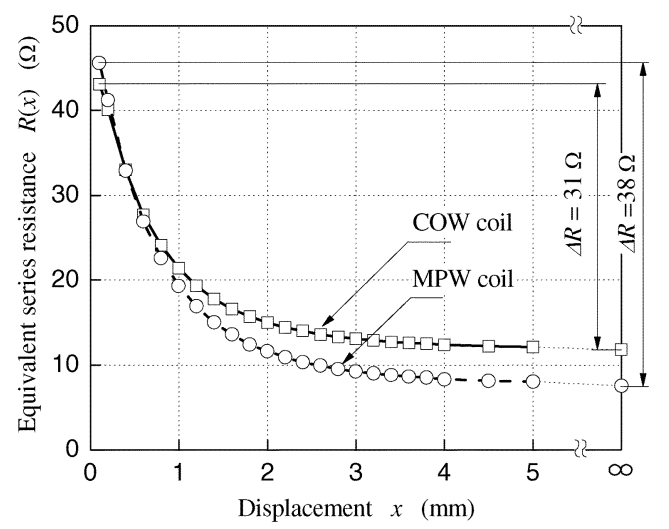

(a)

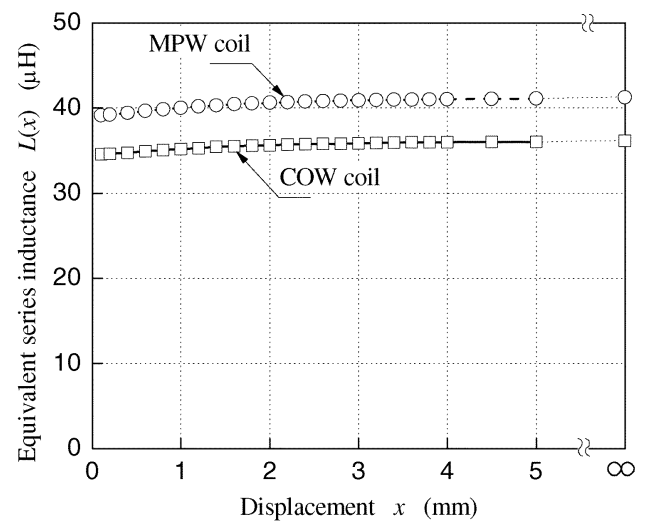

(b)

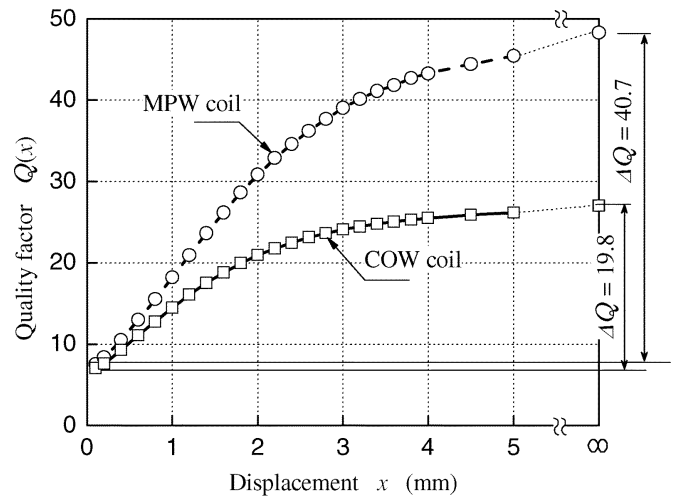

(c)

Fig. 8. Comparison of impedance characteristics between COW and MPW coils versus displacement $x$ at $f=1.4 \mathrm{MHz}$. (a) Resistance $R(x)$. (b) Inductance $L(x)$. (c) Quality factor $Q(x)$.

that of the COW coil. This is because the magnetic thin film of the MPW coil has a reduced proximity effect, as shown in Fig. 5. The inductances $L(\infty)$ of the COW and MPW coils were measured as $37 \mu \mathrm{H}$ and $41 \mu \mathrm{H}$, respectively, in the frequency range from $100 \mathrm{kHz}$ to $2 \mathrm{MHz}$; in other words, the inductance of the MPW coil was 1.1 times that of the COW coil, as shown in Fig. 7(b). As a result of the decrease in $R(\infty)$ and the increase in $L(\infty)$ in the MPW coil, the quality factor $\mathrm{Q}(\infty)$ of the MPW coil was 1.76 times that of the COW coil, as shown in Fig. 7(c).

\section{OutPut Voltage CHARACTERISTICS OF COILS}

\section{A. Impedance Characteristics of Coils}

Fig. 8 shows a comparison of the impedance characteristics between the COW and MPW coils against the displacement.

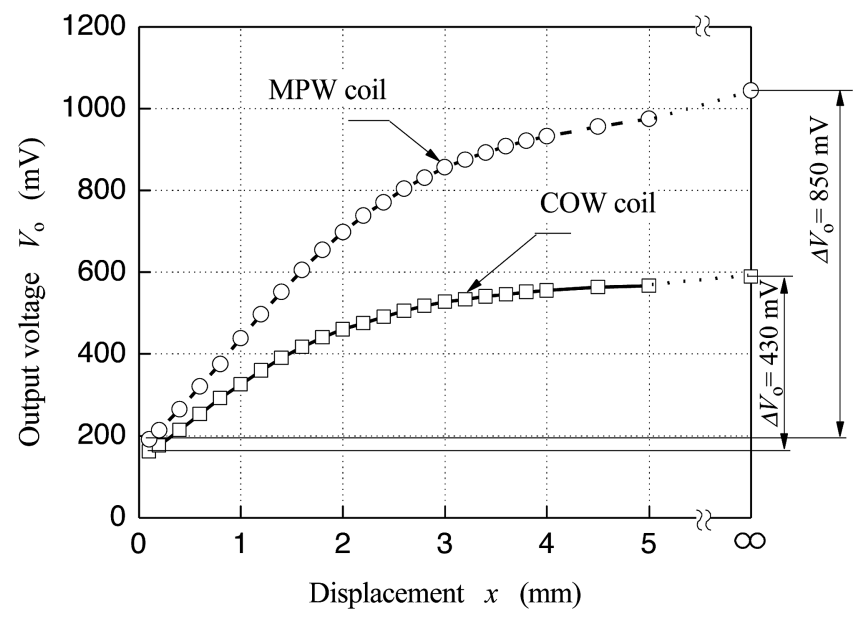

Fig. 9. Comparison of output voltage characteristics between COW and MPW coils at $f=1.4 \mathrm{MHz}$.

The changes in resistance $\Delta R$ of $R(x)$ for the MPW and COW coils were 38 and $31 \Omega$, respectively, as shown in Fig. 8(a), and $\Delta R$ of the MPW coil was 1.2 times that of the COW coil. This difference was caused mainly by the MPW coil generating a larger magnetic flux $\Phi_{c}$ on the surface of the measuring object than that of the COW coil, as shown in Fig. 6. This resulted in an increase in the eddy current loss in the measuring object when the MPW coil was used. Therefore, $\Delta R$ of the MPW coil increases more sharply than that of the COW coil when the displacement $x$ becomes small.

$L(x)$ of the COW and MPW coils showed little change over the range of displacements $x$, as shown in Fig. 8(b). The $L(\infty)$ values of the MPW and the COW coils were 40 and $36 \mu \mathrm{H}$, respectively, and $L(\infty)$ of the MPW coil was 1.1 times that of the COW coil. The increase in $L(x)$ of the MPW coil was due to the magnetic thin films with large permeability.

$\Delta R$ and $L(x)$ of the MPW coil were larger than those of the COW coil, as shown in Fig. 8(a) and (b). Fig. 8(c) shows that $\Delta Q$ of the MPW coil is approximately twice as large as that of the COW coil in the larger displacement range.

\section{B. Output Voltage Characteristics of MPW Coil}

Fig. 9 shows the comparison of the output voltage characteristics of the COW and MPW coils. The changes $\Delta V_{\mathrm{O}}$ of the output voltages of the COW and MPW coils were 430 and $850 \mathrm{mV}$, respectively, and $\Delta V_{\mathrm{O}}$ and $\Delta Q$ of the MPW coil were approximately twice those of the COW coil.

1) Displacement Sensing Sensitivity: Fig. 10 shows the comparison of detection sensitivity between the COW and MPW coils.

The detection sensitivity $\Delta V_{\mathrm{o}}\left(x_{n}\right) / \Delta x_{n}$ is calculated using

$$
\frac{\Delta V_{\mathrm{o}}\left(x_{n}\right)}{\Delta x_{n}}=\frac{V_{\mathrm{o}}\left(x_{n}\right)-V_{\mathrm{o}}\left(x_{n-1}\right)}{x_{n}-x_{n-1}} \quad(\mathrm{~V} / \mathrm{m}) .
$$

The maximum values of detection sensitivity $\Delta V_{\mathrm{o}} / \Delta x$ of the MPW and COW coils were 310 and $195 \mathrm{~V} / \mathrm{m}$, respectively; thus, the detection sensitivity of the MPW coil was 1.5 times that of the COW coil in the entire displacement range. In other words, a $50 \%$ higher displacement sensitivity was obtained with the MPW coil compared with that of the COW coil. 


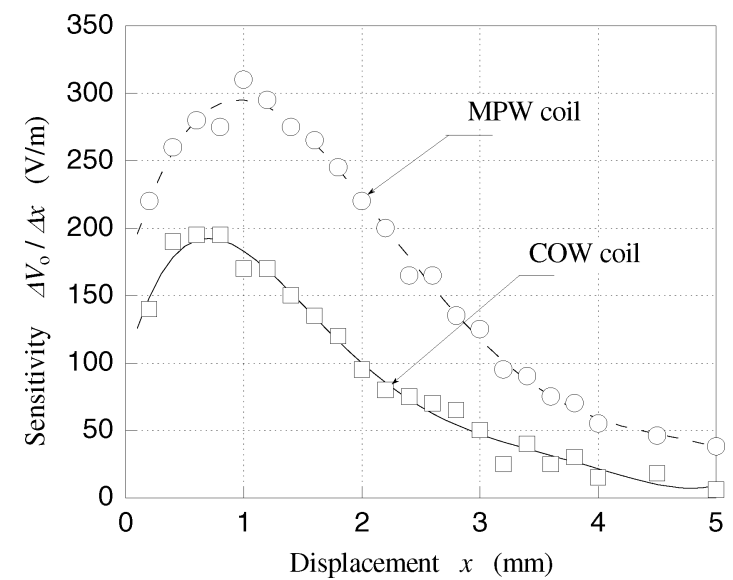

Fig. 10. Comparison of detection sensitivity $\Delta V_{\circ} / \Delta x$ between COW and MPW coils.

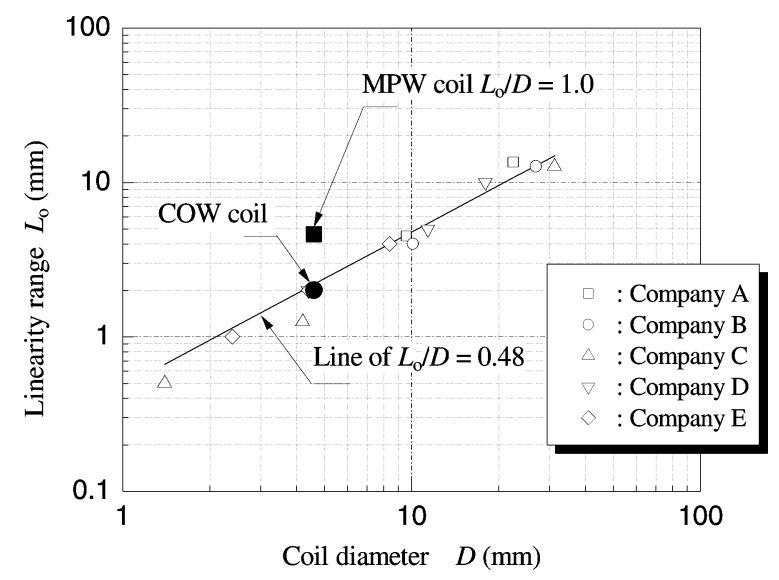

Fig. 11. Linearity range versus coil diameter of EC displacement sensors.

2) Extending Displacement Measuring Range: Because the output characteristic of the coil is generally nonlinear, as shown in Fig. 9, a linearizer is employed to induce output voltage linearity.

The output voltage $V_{\text {out }}$ characteristic was compensated using the linearizer calculated using

$$
\begin{aligned}
V_{\text {out }} & =f_{2}\left(V_{3}\right) & & (\mathrm{V}) \\
V_{3} & =\alpha_{2}\left(V_{2}+v_{2}\right) & & (\mathrm{V}) \\
V_{2} & =f_{1}\left(V_{1}\right) & & (\mathrm{V}) \\
V_{1} & =\alpha_{1}\left(V_{\mathrm{o}}+v_{1}\right) & & (\mathrm{V})
\end{aligned}
$$

where $f_{1}$ and $f_{2}$ are the linearization functions, and $\alpha_{1}, \alpha_{2}, v_{1}$, and $v_{2}$ are constants.

Logarithmic, exponential, polynomial, and the tangent functions are generally used for the linearizer. In this study, we applied a tenth power approximation so that the linearity range of the COW and MPW coils was as wide as possible. In this study, we defined the linearity range $L_{\mathrm{o}}$ as the range in which the difference between the $V_{\text {out }}$ against $x$ characteristic using (10)-(13) and the approximation is less than $\pm 1 \%$.

Fig. 11 shows the linearity range $L_{\mathrm{o}}$ against coil diameter $D$ of EC displacement sensors available from five suppliers: three Japanese, one American, and one European. The $L_{\mathrm{o}} / D$ ratios of the MPW and COW coils plotted on this chart were compensated by the linearizer. The average value of $L_{\mathrm{o}} / D$ for the five suppliers was 0.48 . The $L_{\mathrm{o}} / D$ ratios of the MPW and COW coils were 1.0 and 0.48 , respectively, after linearization.

In conventional technology, $D$ needs to be increased to extend the measuring range. Because the $L_{\mathrm{o}} / D$ ratio of the MPW coil was increased to more than twice that of the average of existing EC displacement sensors, the use of the MPW coil has great potential to extend the measuring range of the EC displacement sensor.

\section{CONCLUSION}

The EC displacement sensor using magnetoplated wire was proposed to extend its measuring range.

\section{A. Physical Phenomena of MPW Coil}

The MPW coil decreases the resistance caused by the proximity effect, increases the inductance, and provides more magnetic flux to the measuring object. These changes were due to the magnetic thin film plated on the wire.

As a result, the quality factor $Q(x)$ of the MPW coil was significantly larger than that of the COW coil, as shown in Fig. 8 .

\section{B. Improvement in Displacement Sensing Sensitivity}

The maximum values of detection sensitivity $\Delta V_{\mathrm{o}} / \Delta x$ of the MPW and COW coils were 310 and $195 \mathrm{~V} / \mathrm{m}$, respectively, and the detection sensitivity of the MPW coil was 1.5 times that of the COW coil in the entire displacement range, as shown in Fig. 10.

\section{Extending Displacement Measuring Range}

The $L_{\mathrm{o}} / D$ ratios of the MPW and COW coils were 1.0 and 0.48 , respectively. The $L_{\mathrm{o}} / D$ ratio of the MPW coil was more than twice those of existing EC displacement sensors. The measuring range of the sensor is expected to be extended when the MPW coil is used, as shown in Fig. 11.

\section{ACKNOWLEDGMENT}

The authors sincerely thank Dr. T. Kunio of Hitachi HighTech. Electronics Engineering Co., Ltd., and Dr. K. Satoshi for discussions.

\section{REFERENCES}

[1] T. Mizuno, T. Hayashi, K. Fumihito, T. Asahina, S. Enoki, H. Sinagawa, H. Yamada, S. Watanabe, S. Uehara, T. Kishimoto, and K. Takeshita, "Eddy current displacement sensor using magnet-plated wire," in IEE Technical Meeting on Magnetics, 2004, vol. MAG-04-185, pp. 19-24.

[2] T. Mizuno, T. Hayashi, K. Fumihito, S. Enoki, H. Sinagawa, H. Yamada, S. Watanabe, T. Kishimoto, and K. Takeshita, "The analysis methods for the quality factor of an eddy current displacement sensor," J. Magn. Soc. Jpn., vol. 29, no. 3, pp. 296-301, 2005.

[3] S. Yoshimura, S. Yoshihara, T. Shirakashi, E. Sato, and K. Ishii, "Characteristics of high-Q coils composed of Fe-plated $\mathrm{Cu}$ wire," in First Magneto Electronics Int. Symp., 1994, pp. 485-487.

[4] N. Ning, X. P. Li, J. Fan, W. C. Ng, Y. P. Xu, X. Qian, and H. L. Seet, "A tunable magnetic inductor," IEEE Trans. Magn., vol. 42, no. 5, pp. 1585-1590, May 2006.

[5] Maxwell 2D Version 8 Ansoft Co., Ltd. 
[6] K. R. Davey and D. Zheng, "Prediction and use of impedance matrices for eddy-current problems," IEEE Trans. Magn., vol. 33, no. 4, pp. 2478-2485, Jul. 1997.

[7] X. Nan and C. R. Sullivan, "Simplified high-accuracy calculation of eddy-current loss in round-wire windings," in Proc. 35th Annu. IEEE Power Electronics Specialist Conf., Jun. 2004, vol. 2, no., pp. 873-879.

[8] J. Acero, R. Alonso, L. A. Barragan, and J. M. Burdio, "Magnetic vector potential based model for eddy-current loss calculation in round-wire planar windings," IEEE Trans. Magn., vol. 42, no. 9, pp. 2152-2158, Sep. 2006.

[9] M. Bartoli, N. Noferi, and A. Reatti, "Modeling winding losses in high-frequency power inductors," J. Circuits, Syst. Comput., vol. 5, no. 4, pp. 607-626, 1995.

[10] N. W. McLachlan, Bessel Functions for Engineers, 2nd ed. Oxford, U.K.: Clarendon, 1954, pp. 137-152.

Manuscript received January 16, 2006; revised November 1, 2006. Corresponding author: T. Mizuno (e-mail: mizunot@gipwc.shinshu-u.ac.jp).

Tsutomu Mizuno was born in Nagano, Japan, in 1958. He received the B.S., M.S., and Ph.D. degrees from Shinshu University, Nagano, Japan, in 1981, 1983, and 1994, respectively.

He joined Amada Co., Isehara, Japan, in 1983. Since 1999, he has been with Shinshu University as an Associate Professor and has engaged in the research and development of magnetic sensors and linear motors.

Dr. Mizuno is a member of the Magnetic Society of Japan and the Institute of Electrical Engineers of Japan.
Shigemi Enoki was born in Hiroshima, Japan, in 1959. He received the B.S. and M.S. degrees from Okayama University of Science, Okayama, Japan, in 1984 and 1986, respectively, and the Ph.D. degree from Shinshu University, Nagano, Japan, in 2003.

He joined Shinkawa Sensor Technology Inc., Higashi-Hiroshima, Japan, in 1994 and has been engaged in the research and development of magnetic displacement sensors.

Dr. Enoki is a member of the Magnetic Society of Japan and the Institute of Electrical Engineers of Japan.

Toshiaki Hayashi was born in Aichi, Japan, in 1981. He received the B.S. degree from Shinshu University, Nagano, Japan, in 2004. He is a graduate student of Shinshu University.

Takashi Asahina was born in Nagano, Japan, in 1983. He received the B.S. degree from Shinshu University, Nagano, Japan, in 2005. He is a graduate student of Shinshu University.

Hiroki Shinagawa was born in Shimane, Japan, in 1975. He received the B.S. degree in electrical engineering from Hiroshima Institute of Technology, Hiroshima, Japan, in 1997.

He joined Shinkawa Electric Co., Ltd., Hiroshima, Japan, in 1997 and has been engaged in research on MEMS devices for accelerometers at Tohoku Gakuin University, Tagajo, Japan. He moved to Shinkawa Sensor Technology, Inc., Higashi-Hiroshima, Japan, in 2004 and is currently engaged in research on eddy-current displacement sensors. 